\title{
Article \\ Brain Structural Connectivity Differences in Patients with Normal Cognition and Cognitive Impairment
}

\author{
Nauris Zdanovskis ${ }^{1,2, *(\mathbb{D}}$, Ardis Platkājis ${ }^{1,2}{ }$, Andrejs Kostiks ${ }^{3}$, Guntis Karelis ${ }^{3}$ and Olesja Grigorjeva ${ }^{4}$ \\ 1 Department of Radiology, Riga Stradins University, Dzirciema Street 16, LV-1007 Riga, Latvia; \\ ardis.platkajis@rsu.lv \\ 2 Department of Radiology, Riga East University Hospital, Hipokrata Street 2, LV-1038 Riga, Latvia \\ 3 Department of Neurosurgery and Neurology, Riga East University Hospital, Hipokrata Street 2, \\ LV-1038 Riga, Latvia; andrejs.kostiks@gmail.com (A.K.); guntis.karelis@gmail.com (G.K.) \\ 4 Department of Computer Control Systems, Riga Technical University, Kalıu Street 1, LV-1658 Riga, Latvia; \\ Olesja.Grigorjeva@rtu.lv \\ * Correspondence: nauris.zdanovskis@rsu.lv
}

Citation: Zdanovskis, N.; Platkājis, A.; Kostiks, A.; Karelis, G.; Grigorjeva, O. Brain Structural Connectivity Differences in Patients with Normal Cognition and Cognitive Impairment. Brain Sci. 2021, 11, 943. https:// doi.org/10.3390/brainsci11070943

Academic Editor: Carlo

Cosimo Quattrocchi

Received: 14 June 2021

Accepted: 15 July 2021

Published: 18 July 2021

Publisher's Note: MDPI stays neutral with regard to jurisdictional claims in published maps and institutional affiliations.

Copyright: (c) 2021 by the authors. Licensee MDPI, Basel, Switzerland. This article is an open access article distributed under the terms and conditions of the Creative Commons Attribution (CC BY) license (https:// creativecommons.org/licenses/by/ $4.0 /)$.

\begin{abstract}
Advances in magnetic resonance imaging, particularly diffusion imaging, have allowed researchers to analyze brain connectivity. Identification of structural connectivity differences between patients with normal cognition, cognitive impairment, and dementia could lead to new biomarker discoveries that could improve dementia diagnostics. In our study, we analyzed 22 patients (11 control group patients, 11 dementia group patients) that underwent 3T MRI diffusion tensor imaging (DTI) scans and the Montreal Cognitive Assessment (MoCA) test. We reconstructed DTI images and used the Desikan-Killiany-Tourville cortical parcellation atlas. The connectivity matrix was calculated, and graph theoretical analysis was conducted using DSI Studio. We found statistically significant differences between groups in the graph density, network characteristic path length, small-worldness, global efficiency, and rich club organization. We did not find statistically significant differences between groups in the average clustering coefficient and the assortativity coefficient. These statistically significant graph theory measures could potentially be used as quantitative biomarkers in cognitive impairment and dementia diagnostics.
\end{abstract}

Keywords: DTI; MRI; brain connectivity; MCI; dementia; mild cognitive impairment; neurodegenerative diseases

\section{Introduction}

In recent years, scientists have been able to study the brain in ways they never could before, due to advancements in imaging technologies. One of these advances is diffusion tensor imaging (DTI), which tracks water movement through the brain's white matter [1]. DTI has allowed researchers to analyze DTI metrics and brain connectivity differences between patients with cognitive impairment and normal cognition [2,3]. Brain connectivity plays an important role in sustaining the function of the human brain and cognition. Thus, analyzing brain connectivity could potentially lead us to imaging biomarker discoveries that could aid in cognitive impairment diagnostics $[4,5]$.

It is important to distinguish between functional and structural connectivity. Functional connectivity can be tracked, measured, and analyzed using functional methods, i.e., functional magnetic resonance imaging (fMRI), positron emission tomography (PET), electroencephalography (EEG), magnetoencephalography (MEG), and others [6,7].

On the other hand, white matter bundles and tracts can be visualized by utilizing diffusion imaging on magnetic resonance machines, particularly diffusion tensor imaging (DTI). By utilizing DTI, it is possible to visualize white matter tracts, reconstruct them, and apply graph theory measures to analyze differences in these tracts among individuals [8]. These measurements can be used in specific brain regions (specific white matter tracts) or could be used in a whole-brain connectometry analysis [9]. 
To perform whole-brain connectometry analysis, it is necessary to define which cortical atlas would be used for brain parcellation. Several available cortical parcellation atlases are used in neuroimaging and network analysis, i.e., the Desikan-Killiany-Tourville (DKT) atlas [10], the automated anatomical labeling 3 (AAL3) atlas [11], Brainnetome Atlas [12], and others. In our study, we used DKT cortical parcellation.

In graph theory, numerous measures could be used as a quantitative variable to assess brain connectivity patterns, for example, measures of integration (degree level, shortest path length, number of triangles, characteristic path length, global efficiency, clustering coefficient, transitivity, local efficiency, modularity), measures of centrality (closeness centrality, betweenness centrality, within-module degree, participation coefficient), network motifs (anatomical and functional motifs, motif z-score, motif fingerprint), measures of resilience (degree distribution, average neighbor degree, assortativity coefficient), and other measures [13].

In our study, we analyzed undirected and unweighted graph relationships and included:

1. Graph density (the mean network degree);

2. Average clustering coefficient (fraction of triangles around an individual node);

3. Network characteristic path length (the average shortest path length between all pairs of nodes in the network);

4. Small-worldness (small-world networks are significantly more clustered than random networks, yet have approximately the same characteristic path length as random networks);

5. Global efficiency (average inverse shortest path length);

6. Assortativity coefficient (correlation coefficient between the degrees of all nodes on two opposite ends of a link);

7. Rich club coefficient, $\mathrm{k}=5 ; 10 ; 15 ; 20$ (network high-degree nodes that, on average, are more intensely interconnected than lower-degree nodes).

The goal of this research was to identify the changes in structural connectivity between the control group and the dementia group.

\section{Materials and Methods}

\subsection{Participants}

Participants were admitted to a neurologist with suspected cognitive impairment. All participants were evaluated by a board-certified neurologist, and the Montreal Cognitive Assessment (MoCA) was performed.

All participants in the control group and dementia group had at least 16 years of education. For the control group, we used MoCA cutoff scores of $\geq 23$, and for the dementia group, we used a cutoff of $\leq 22$, as these values can produce a high sensitivity, specificity, and AUC [14,15].

Based on the neurological assessment and MoCA test results, patients were divided into two groups: the control group (patients with no severe cognitive impairment), and the dementia group (patients with cognitive impairment who need supervision or some help during daily activities).

The patient demographic data and MoCA scores are shown in Table 1.

Table 1. Patient age and MoCA scores in study groups (D-dementia group, $\mathrm{C}$-control group).

\begin{tabular}{ccccc}
\hline & \multicolumn{2}{c}{ MoCA } & \multicolumn{2}{c}{ Age } \\
\cline { 2 - 5 } & D & C & D & C \\
\hline Participants & 11 & 11 & 11 & 11 \\
Mean & 12.818 & 26.182 & 75.000 & 62.273 \\
Median & 13 & 25 & 71 & 69 \\
Std. Deviation & 5.036 & 2.750 & 10.488 & 15.395 \\
Minimum & 4 & 23 & 65 & 35 \\
Maximum & 20 & 30 & 96 & 77 \\
\hline
\end{tabular}


Exclusion criteria for study patients were clinically significant neurological diseases (tumors, major stroke, malformations, etc.), drug use, and alcohol abuse. All study patients did not have any other significant abnormalities on magnetic resonance (MR) scans.

Based on the neurological assessment and MRI scans, all patients had a mixed type of dementia.

\subsection{Magnetic Resonance Imaging (MRI) Data Acquisition and Image Reconstruction}

MRI was performed at a single-site university hospital to avoid inter-scanner differences. All scans were converted from DICOM format to Neuroimaging Informatics Technology Initiative (NIfTI) format, and further to SRC and FIB format to perform tractography analysis.

The diffusion images were acquired on a GE SIGNA Architect 3T scanner using a diffusion sequence $(\mathrm{TE}=101.5 \mathrm{~ms}, \mathrm{TR}=14,884 \mathrm{~ms})$. A DTI diffusion scheme was used, and a total of 30 diffusion sampling directions were acquired. The b-value was $1000.59 \mathrm{~s} / \mathrm{mm}^{2}$. The in-plane resolution was $0.9375 \mathrm{~mm}$. The slice thickness was $2 \mathrm{~mm}$. The b-table was checked by an automatic quality control routine to ensure its accuracy [16]. The b-table was flipped by $0.012 \mathrm{fz}$. The restricted diffusion was quantified using restricted diffusion imaging [17]. The diffusion data were reconstructed using generalized q-sampling imaging [18], with a diffusion sampling length ratio of 1.25. A deterministic fiber tracking algorithm [19] was used with augmented tracking strategies [20] to improve reproducibility. A seeding region was placed at the whole brain. The anisotropy threshold was randomly selected. The angular threshold was randomly selected from 15 degrees to 90 degrees. The step size was randomly selected from 0.5 to 1.5 voxels. Tracks with a length shorter than 30 or longer than $300 \mathrm{~mm}$ were discarded. A total of 10,000,000 seeds were placed.

Free Surfer DKT was used as the brain parcellation, and the connectivity matrix was calculated by using the count of the connecting tracks. The connectivity matrix and graph theoretical analyses were conducted using DSI Studio (http:/ / dsi-studio.labsolver.org, accessed on 3 may 2021) [21].

Acquired images after tractography reconstruction, fiber tracking, and connectivity matrix analysis are shown in Figure 1.

\subsection{Statistical Analysis}

Data were analyzed using statistical analysis software JASP Version 0.14.1. The MannWhitney $U$ test was used to determine statistically significant differences between the control group and the dementia group. Results with a $p$-value smaller than 0.05 were considered statistically significant. Descriptive statistics for statistically significant network measures were calculated. 


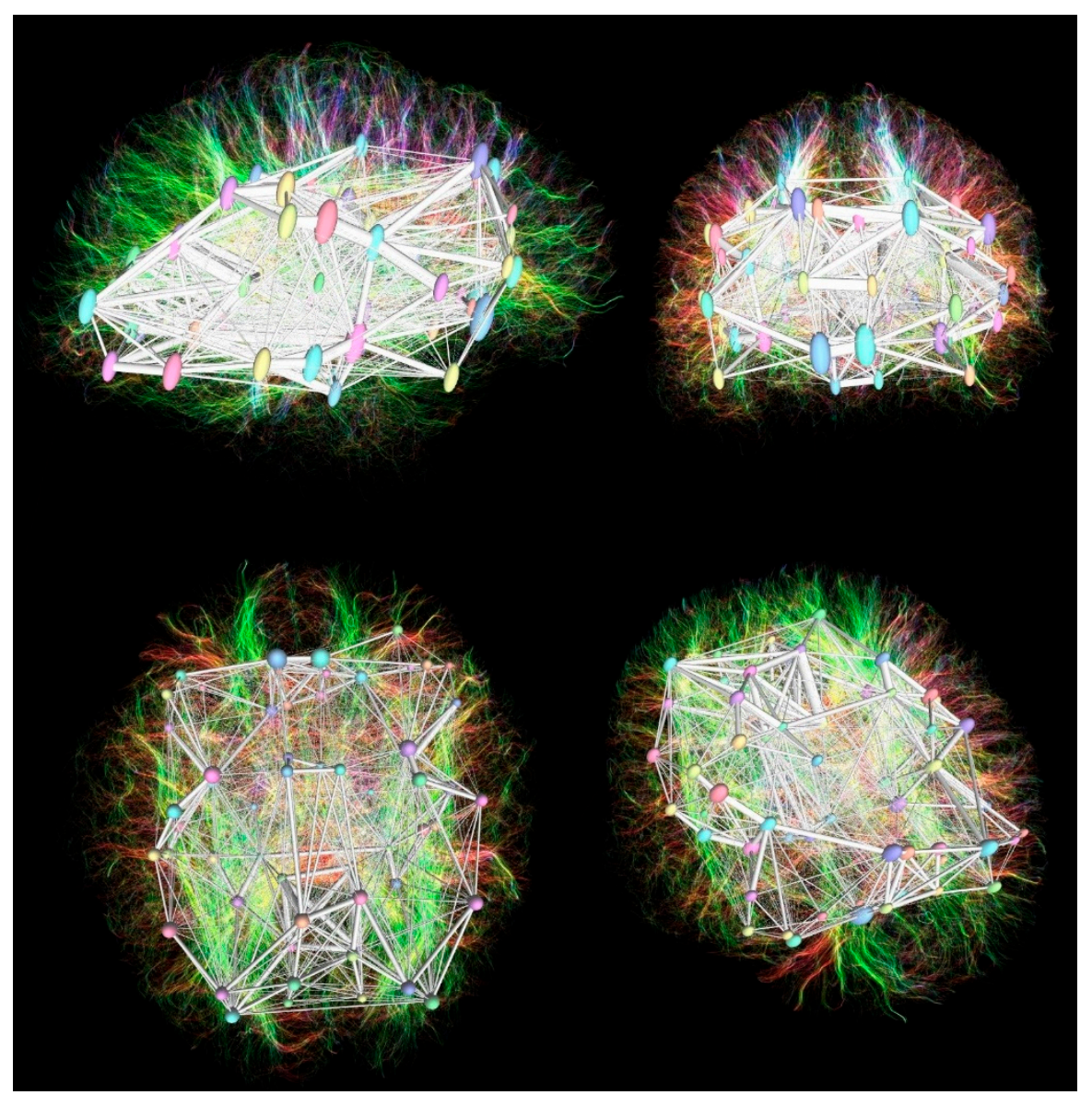

Figure 1. The control group patients' DTI tractography with overlayed DKT cortical parcellation regions and connectivity matrix reconstruction in sagittal (upper left), coronal (upper right), axial (lower left), and oblique (lower right) projections.

\section{Results}

In total, 22 patients were included in this study: 11 patients in the control group ( 7 females, 4 males; average age 62.2 years, standard deviation 15.4, median age 69, minimum 35 , maximum 77), and 11 patients in the dementia group ( 7 females, 4 males, average age 75.0 years, standard deviation 10.5 , median age 71 , minimum 65 , maximum 96).

The Mann-Whitney $U$ test results between study groups are shown in Table 2.

Table 2. Mann-Whitney U test comparing control group and dementia group.

\begin{tabular}{ccc}
\hline & Mann-Whitney U Test & \\
\hline & W & $p$ \\
\hline Graph Density & 27.000 & $0.030^{*}$ \\
Average Clustering Coefficient & 34.000 & 0.088 \\
Network Characteristic Path Length & 94.000 & $0.028^{*}$ \\
Small-Worldness & 30.000 & $0.047^{*}$ \\
Global Efficiency & 26.000 & $0.023^{*}$ \\
Assortativity Coefficient & 69.000 & 0.606 \\
Rich Club (k=5) & 27.000 & $0.030^{*}$ \\
Rich Club (k=10) & 29.000 & $0.042^{*}$ \\
Rich Club (k=15) & 27.000 & $0.028^{*}$ \\
Rich Club (k=20) & 30.000 & $0.047^{*}$ \\
\hline
\end{tabular}


We found statistically significant differences between groups in the graph density, network characteristic path length, small-worldness, global efficiency, and rich club coefficient $(k=5 ; 10 ; 15 ; 20)$. We did not find statistically significant differences between groups in the average clustering coefficient and the assortativity coefficient. Additionally, we analyzed descriptive statistics for statistically significant measures.

\subsection{Graph Density}

The graph density is the fraction of present connections to possible connections [13]. Our findings on the graph density are shown in Table 3. The graph density was higher in control group patients.

Table 3. Graph density descriptive statistics in dementia group (D) and control group (C).

\begin{tabular}{ccc}
\hline & \multicolumn{3}{c}{ Graph Density } \\
\hline & D & C \\
\hline Mean & 0.621 & 0.679 \\
Std. Error of Mean & 0.017 & 0.018 \\
Median & 0.625 & 0.687 \\
Std. Deviation & 0.057 & 0.059 \\
Minimum & 0.537 & 0.563 \\
Maximum & 0.689 & 0.753 \\
\hline
\end{tabular}

\subsection{Average Clustering Coefficient}

The clustering coefficient represents the degree to which nodes in a graph tend to cluster together [22]. Our findings on the average clustering coefficient are shown in Table 4 . The average clustering coefficient was higher in the control group patients.

Table 4. Average clustering coefficient descriptive statistics in dementia group (D) and control group (C).

\begin{tabular}{ccc}
\hline & \multicolumn{2}{c}{ Average Clustering Coefficient } \\
\hline D & C \\
\hline Mean & 0.807 & 0.825 \\
Std. Error of Mean & 0.008 & 0.011 \\
Median & 0.802 & 0.832 \\
Std. Deviation & 0.027 & 0.036 \\
Minimum & 0.759 & 0.739 \\
Maximum & 0.841 & 0.860 \\
\hline
\end{tabular}

\subsection{Network Characteristic Path Length}

The network characteristic path length (or average path length) is defined as the average distance between all pairs of vertices [23]. Our findings on the network characteristic path length are shown in Table 5. The network characteristic path length was shorter in control group patients.

\subsection{Small-Worldness}

A small-world network is a graph where most nodes are not neighbors, but the neighbors of any given node are likely to be neighbors of each other, and most nodes can be reached from every other node by a small number of edges [24]. Our findings on the small-worldness coefficient are shown in Table 6. The small-worldness coefficient was higher in control group patients. 
Table 5. Network characteristic path length descriptive statistics in dementia group (D) and control group (C).

\begin{tabular}{ccc}
\hline & \multicolumn{2}{c}{ Network Characteristic Path Length } \\
\hline & D & C \\
\hline Mean & 1.393 & 1.334 \\
Std. Error of Mean & 0.018 & 0.018 \\
Median & 1.388 & 1.324 \\
Std. Deviation & 0.059 & 0.059 \\
Minimum & 1.330 & 1.260 \\
Maximum & 1.483 & 1.448 \\
\hline
\end{tabular}

Table 6. Small-worldness descriptive statistics in dementia group (D) and control group (C).

\begin{tabular}{ccc}
\hline & \multicolumn{3}{c}{ Small-Worldness } \\
\hline D & C \\
\hline Mean & 0.580 & 0.620 \\
Std. Error of Mean & 0.012 & 0.016 \\
Median & 0.589 & 0.624 \\
Std. Deviation & 0.041 & 0.051 \\
Minimum & 0.511 & 0.510 \\
Maximum & 0.633 & 0.682 \\
\hline
\end{tabular}

\subsection{Global Efficiency}

Global efficiency is defined as the average inverse shortest path length in the network [25]. Our findings on global efficiency are shown in Table 7. Global efficiency was higher in control group patients.

Table 7. Global efficiency descriptive statistics in dementia group (D) and control group (C).

\begin{tabular}{ccc}
\hline & \multicolumn{3}{c}{ Global Efficiency } \\
\hline D & C \\
\hline Mean & 0.797 & 0.826 \\
Std. Error of Mean & 0.009 & 0.009 \\
Median & 0.798 & 0.830 \\
Std. Deviation & 0.029 & 0.029 \\
Minimum & 0.754 & 0.769 \\
Maximum & 0.830 & 0.862 \\
\hline
\end{tabular}

\subsection{Rich Club}

Rich club regions are a set of highly interconnected nodes forming a tight subnetwork within a network. In rich club analysis, $k$ represents the number of connections attached to each network node [26]. In our analysis, we used k values of 5, 10, 15, and 20. In all cases, there were statistically significant differences between groups, and higher results were found in control group patients. Our findings on rich club organization are shown in Table 8. 
Table 8. Rich club descriptive statistics with different $k$ values in the dementia group (D) and control group (C).

\begin{tabular}{ccccccccc}
\hline & \multicolumn{2}{c}{ Rich Club(k= 5) } & \multicolumn{2}{c}{ Rich Club(k= 10) } & \multicolumn{2}{c}{ Rich Club(k= 15) } & \multicolumn{2}{c}{ Rich Club(k=20) } \\
\hline & D & C & D & C & D & C & D & C \\
\hline Mean & 0.621 & 0.679 & 0.628 & 0.685 & 0.657 & 0.702 & 0.704 & 0.736 \\
Std. Error of Mean & 0.017 & 0.018 & 0.017 & 0.017 & 0.014 & 0.016 & 0.011 & 0.015 \\
Median & 0.625 & 0.687 & 0.625 & 0.687 & 0.677 & 0.714 & 0.702 & 0.753 \\
Std. Deviation & 0.057 & 0.059 & 0.057 & 0.058 & 0.046 & 0.054 & 0.035 & 0.051 \\
Minimum & 0.537 & 0.563 & 0.538 & 0.563 & 0.558 & 0.597 & 0.656 & 0.619 \\
Maximum & 0.689 & 0.753 & 0.689 & 0.753 & 0.705 & 0.753 & 0.764 & 0.796 \\
\hline
\end{tabular}

\section{Discussion}

There are different approaches on how to analyze structural connectivity in brain. It is possible to analyze whole-brain connectivity patterns or focus on specific brain regions and DTI metrics (fractional anisotropy, mean diffusivity, number of streamlines etc.) $[27,28]$. In our study, we performed whole-brain connectometry analysis and compared graph theory measures between patients with normal cognition and patients with cognitive impairment. We used the DKT cortical parcellation atlas to estimate graph theory values and quantitative measurements.

In general, connectometry analysis in patients with cognitive impairment could be a promising diagnostic method that provides quantitative biomarkers [29].

Our results on global efficiency are consistent with other studies that compared brain network global efficiency and cognitive abilities, i.e., global efficiency was a significant predictor of working memory performance [25]. Lower global efficiency values are a significant predictor factor in conversion to dementia in patients with small vessel disease [30].

In our study, the characteristic path length was higher in patients with cognitive impairment, which is consistent with Alzheimer's dementia (AD)-related changes [31], and was found to be consistent with a functional network study where a longer path length was observed in AD patients [32].

In our study, the small-worldness coefficient was lower in patients with cognitive impairment. There are reports where patients with $\mathrm{AD}$ tend to lose small-worldness properties [31-33].

There were statistically significant differences in rich club organization with all measured coefficients $(k=5,10,15,20)$, with higher values in the control group. Rich club organization is very important for global brain communication and global integration of information [34,35]. Rich club disruption could be connected to early-onset AD [36], and, in general, it is associated with MCI and AD-related changes in the brain [37,38].

Although we did not find statistically significant differences between groups in the clustering coefficient, in other research papers, it was stated that in patients with $\mathrm{AD}$, the clustering coefficient is higher than in control subjects [31].

\section{Limitations}

This was an exploratory research study with a limited patient cohort. To confirm the theses stated in this article, we intend to continue the research and validate the results on a larger patient cohort.

\section{Conclusions}

In our study, the graph density, network characteristic path length, small-worldness, global efficiency, and rich club coefficient showed statistically significant differences between control and dementia patient groups.

Thus, these graph theory measures could potentially be used as quantitative biomarkers in cognitive impairment and dementia diagnostics.

Further studies with a larger patient cohort should evaluate and validate the diagnostic certainty and prognostic value of these graph theory measures. 
Author Contributions: Conceptualization, N.Z., A.P.; methodology, N.Z., A.P., A.K., G.K., O.G.; software, N.Z., O.G.; validation, A.P., G.K.; formal analysis, N.Z., A.P., G.K.; investigation, A.K., G.K.; resources, A.P., G.K.; data curation, N.Z.; writing-original draft preparation, N.Z.; writingreview and editing, N.Z., A.P., A.K., G.K., O.G.; visualization, N.Z.; supervision, A.P., G.K.; project administration, N.Z. All authors have read and agreed to the published version of the manuscript.

Funding: This research received no external funding.

Institutional Review Board Statement: This study was conducted according to the guidelines of the Declaration of Helsinki and approved by the Institutional Review Board of Riga East University Hospital (No. AP-144/10), and the ethics committee at Riga East University Hospital Ethics Board (No. 08-A/19).

Informed Consent Statement: Informed consent was obtained from all subjects involved in the study.

Data Availability Statement: The data presented in this study are available on request from the corresponding author. The data are not publicly available due to data privacy.

Conflicts of Interest: The authors declare no conflict of interest.

\section{References}

1. Alexander, A.L.; Lee, J.E.; Lazar, M.; Field, A.S. Diffusion Tensor Imaging of the Brain. Neurotherapeutics 2007, 4, 316-329. [CrossRef]

2. Mayo, C.D.; Garcia-Barrera, M.A.; Mazerolle, E.L.; Ritchie, L.J.; Fisk, J.D.; Gawryluk, J.R. Relationship Between DTI Metrics and Cognitive Function in Alzheimer's Disease. Front. Aging Neurosci. 2019, 10. [CrossRef]

3. Pandya, S.; Kuceyeski, A.; Raj, A. Alzheimer's Disease Neuroimaging Initiative The Brain's Structural Connectome Mediates the Relationship between Regional Neuroimaging Biomarkers in Alzheimer's Disease. J. Alzheimers Dis. 2017, 55, 1639-1657. [CrossRef]

4. Akil, H.; Martone, M.E.; Van Essen, D.C. Challenges and Opportunities in Mining Neuroscience Data. Science 2011, 331, 708-712. [CrossRef] [PubMed]

5. Yeh, F.-C.; Tang, P.-F.; Tseng, W.-Y.I. Diffusion MRI Connectometry Automatically Reveals Affected Fiber Pathways in Individuals with Chronic Stroke. Neuroimage Clin. 2013, 2, 912-921. [CrossRef] [PubMed]

6. Michel, C.M.; Murray, M.M. Towards the Utilization of EEG as a Brain Imaging Tool. NeuroImage 2012, 61, 371-385. [CrossRef] [PubMed]

7. Raichle, M.E. Functional Brain Imaging and Human Brain Function. J. Neurosci. 2003, 23, 3959-3962. [CrossRef]

8. Farahani, F.V.; Karwowski, W.; Lighthall, N.R. Application of Graph Theory for Identifying Connectivity Patterns in Human Brain Networks: A Systematic Review. Front. Neurosci. 2019, 13. [CrossRef]

9. Sporns, O. Graph Theory Methods: Applications in Brain Networks. Dialogues Clin. Neurosci. 2018, 20, 111-121.

10. Klein, A.; Tourville, J. 101 Labeled Brain Images and a Consistent Human Cortical Labeling Protocol. Front. Neurosci. 2012, 6. [CrossRef]

11. Rolls, E.T.; Huang, C.-C.; Lin, C.-P.; Feng, J.; Joliot, M. Automated Anatomical Labelling Atlas 3. NeuroImage 2020, $206,116189$. [CrossRef]

12. Fan, L.; Li, H.; Zhuo, J.; Zhang, Y.; Wang, J.; Chen, L.; Yang, Z.; Chu, C.; Xie, S.; Laird, A.R.; et al. The Human Brainnetome Atlas: A New Brain Atlas Based on Connectional Architecture. Cereb. Cortex 2016, 26, 3508-3526. [CrossRef] [PubMed]

13. Rubinov, M.; Sporns, O. Complex Network Measures of Brain Connectivity: Uses and Interpretations. NeuroImage 2010, 52, 1059-1069. [CrossRef] [PubMed]

14. Goldstein, F.C.; Ashley, A.V.; Miller, E.; Alexeeva, O.; Zanders, L.; King, V. Validity of the Montreal Cognitive Assessment as a Screen for Mild Cognitive Impairment and Dementia in African Americans. J. Geriatr. Psychiatry Neurol. 2014, 27, 199-203. [CrossRef] [PubMed]

15. Thomann, A.E.; Berres, M.; Goettel, N.; Steiner, L.A.; Monsch, A.U. Enhanced Diagnostic Accuracy for Neurocognitive Disorders: A Revised Cut-off Approach for the Montreal Cognitive Assessment. Alzheimers Res. Ther. 2020, 12, 39. [CrossRef] [PubMed]

16. Schilling, K.G.; Yeh, F.-C.; Nath, V.; Hansen, C.; Williams, O.; Resnick, S.; Anderson, A.W.; Landman, B.A. A Fiber Coherence Index for Quality Control of B-Table Orientation in Diffusion MRI Scans. Magn. Reson. Imaging 2019, 58, 82-89. [CrossRef] [PubMed]

17. Yeh, F.-C.; Liu, L.; Hitchens, T.K.; Wu, Y.L. Mapping Immune Cell Infiltration Using Restricted Diffusion MRI. Magn. Reson. Med. 2017, 77, 603-612. [CrossRef]

18. Yeh, F.-C.; Wedeen, V.J.; Tseng, W.-Y.I. Generalized Q-Sampling Imaging. IEEE Trans. Med. Imaging 2010, 29, 1626-1635. [CrossRef]

19. Yeh, F.-C.; Verstynen, T.D.; Wang, Y.; Fernández-Miranda, J.C.; Tseng, W.-Y.I. Deterministic Diffusion Fiber Tracking Improved by Quantitative Anisotropy. PLoS ONE 2013, 8, e80713. [CrossRef]

20. Yeh, F.-C. Shape Analysis of the Human Association Pathways. Neuroimage 2020, 223, 117329. [CrossRef] 
21. Yeh, F.-C.; Panesar, S.; Fernandes, D.; Meola, A.; Yoshino, M.; Fernandez-Miranda, J.C.; Vettel, J.M.; Verstynen, T. PopulationAveraged Atlas of the Macroscale Human Structural Connectome and Its Network Topology. Neuroimage 2018, 178, 57-68. [CrossRef] [PubMed]

22. Jungck, J.R.; Viswanathan, R. Chapter 1-Graph Theory for Systems Biology: Interval Graphs, Motifs, and Pattern Recognition. In Algebraic and Discrete Mathematical Methods for Modern Biology; Robeva, R.S., Ed.; Academic Press: Boston, MA, USA, 2015; pp. 1-27, ISBN 978-0-12-801213-0.

23. Junker, B.H.; Schreiber, F. Analysis of Biological Networks; John Wiley \& Sons: Hoboken, NJ, USA, 2011; ISBN 978-1-118-20991-2.

24. Watts, D.J.; Strogatz, S.H. Collective Dynamics of 'Small-World' Networks. Nature 1998, 393, 440-442. [CrossRef] [PubMed]

25. Stanley, M.L.; Simpson, S.L.; Dagenbach, D.; Lyday, R.G.; Burdette, J.H.; Laurienti, P.J. Changes in Brain Network Efficiency and Working Memory Performance in Aging. PLoS ONE 2015, 10. [CrossRef] [PubMed]

26. Kim, D.-J.; Min, B.-K. Rich-Club in the Brain's Macrostructure: Insights from Graph Theoretical Analysis. Comput. Struct. Biotechnol. J. 2020, 18, 1761-1773. [CrossRef]

27. Mallio, C.A.; Schmidt, R.; de Reus, M.A.; Vernieri, F.; Quintiliani, L.; Curcio, G.; Beomonte Zobel, B.; Quattrocchi, C.C.; van den Heuvel, M.P. Epicentral Disruption of Structural Connectivity in Alzheimer's Disease. CNS Neurosci Ther 2015, 21, 837-845. [CrossRef] [PubMed]

28. Zhou, J.; Gennatas, E.D.; Kramer, J.H.; Miller, B.L.; Seeley, W.W. Predicting Regional Neurodegeneration from the Healthy Brain Functional Connectome. Neuron 2012, 73, 1216-1227. [CrossRef]

29. Yeh, F.-C.; Vettel, J.M.; Singh, A.; Poczos, B.; Grafton, S.T.; Erickson, K.I.; Tseng, W.-Y.I.; Verstynen, T.D. Quantifying Differences and Similarities in Whole-Brain White Matter Architecture Using Local Connectome Fingerprints. PLoS Comput. Biol. 2016, 12, e1005203. [CrossRef]

30. Tuladhar, A.M.; van Uden, I.W.M.; Rutten-Jacobs, L.C.A.; Lawrence, A.; van der Holst, H.; van Norden, A.; de Laat, K.; van Dijk, E.; Claassen, J.A.H.R.; Kessels, R.P.C.; et al. Structural Network Efficiency Predicts Conversion to Dementia. Neurology 2016, 86, 1112-1119. [CrossRef]

31. He, Y.; Chen, Z.; Evans, A. Structural Insights into Aberrant Topological Patterns of Large-Scale Cortical Networks in Alzheimer's Disease. J. Neurosci. 2008, 28, 4756-4766. [CrossRef]

32. Stam, C.J.; Jones, B.F.; Nolte, G.; Breakspear, M.; Scheltens, P. Small-World Networks and Functional Connectivity in Alzheimer's Disease. Cereb. Cortex 2007, 17, 92-99. [CrossRef]

33. Sanz-Arigita, E.J.; Schoonheim, M.M.; Damoiseaux, J.S.; Rombouts, S.A.R.B.; Maris, E.; Barkhof, F.; Scheltens, P.; Stam, C.J. Loss of 'Small-World' Networks in Alzheimer's Disease: Graph Analysis of FMRI Resting-State Functional Connectivity. PLoS ONE 2010, 5, e13788. [CrossRef]

34. van den Heuvel, M.P.; Kahn, R.S.; Goñi, J.; Sporns, O. High-Cost, High-Capacity Backbone for Global Brain Communication. Proc. Natl. Acad. Sci. USA 2012, 109, 11372-11377. [CrossRef]

35. van den Heuvel, M.P.; Sporns, O. Rich-Club Organization of the Human Connectome. J. Neurosci. 2011, 31, 15775-15786. [CrossRef] [PubMed]

36. Daianu, M.; Mezher, A.; Mendez, M.F.; Jahanshad, N.; Jimenez, E.E.; Thompson, P.M. Disrupted Rich Club Network in Behavioral Variant Frontotemporal Dementia and Early-onset Alzheimer's Disease. Hum. Brain Mapp. 2015, 37, 868-883. [CrossRef] [PubMed]

37. Dai, Z.; Yan, C.; Li, K.; Wang, Z.; Wang, J.; Cao, M.; Lin, Q.; Shu, N.; Xia, M.; Bi, Y.; et al. Identifying and Mapping Connectivity Patterns of Brain Network Hubs in Alzheimer's Disease. Cereb. Cortex 2015, 25, 3723-3742. [CrossRef]

38. Navas, A.; Papo, D.; Boccaletti, S.; Del-Pozo, F.; Bajo, R.; Maestú, F.; Martínez, J.H.; Gil, P.; Sendiña-Nadal, I.; Buldú, J.M. Functional Hubs in Mild Cognitive Impairment. Int. J. Bifurc. Chaos 2015, 25, 1550034. [CrossRef] 